\title{
Análise Custo-Benefício do Reforço Negativo em Contingências de Esquiva Sinalizada
}

\author{
Carlos Eduardo Cameschi ${ }^{1}$ \\ Universidade de Brasília \\ João Claudio Todorov² \\ Universidade de Brasília e Universidade Católica de Goiás
}

\begin{abstract}
RESUMO - Este estudo analisa as funções dos sinais em contingências de esquiva e descreve a interação organismo-ambiente como exemplo de relação custo-benefício. Seis ratos foram expostos a um procedimento de esquiva sinalizada, onde um período seguro (PS) de $20 \mathrm{~s}$ era seguido pelo período de aviso (PA) de $10 \mathrm{~s}$ e, na ausência de respostas, um choque era liberado e reiniciava o processo. Em sucessivas condições experimentais, o número de respostas requerido durante o PA para cancelar o choque aumentou de (1) um para 10 (FR1, 3, 5, 7 e 10), mantendo-se FR1 durante o PS. Observou-se que a taxa de respostas no PA expressa uma função U invertido em relação aos esquemas FR programados, a taxa de respostas no PS e a taxa de choques liberados expressam uma função direta em relação ao tamanho da FR e a taxa de sinais (número de vezes que o sinal foi apresentado dividido pela duração do PS) expressa uma função inversa em relação à razão requerida durante o PA. Estes resultados são consistentes com uma análise custo-benefício do desempenho em esquiva sinalizada: sendo requeridas poucas respostas durante o sinal, os sujeitos tendiam a responder menos no PS, minimizando esforço; e devido ao aumento progressivo no custo da resposta durante o PA, tornou-se menor o esforço pelo mesmo benefício responder durante o PS.
\end{abstract}

Palavras-chave: reforço negativo; esquiva sinalizada; relação custo-benefício.

\section{A Cost-Benefit Analysis of Responding in Signaled Free-Operant Avoidance of Shock}

\begin{abstract}
This study examined the role that signals have in signaled-avoidance contingencies and analyzed the environment-organism relationship as an example of a cost-benefit relation. Six rats served as subjects in a signaled free-operant procedure with a response-signal interval of $20 \mathrm{~s}$ (safe period) and a signal-shock interval of $10 \mathrm{~s}$ (warning period). In successive experimental conditions the response requirement during the signal was increased from 1 to 10 (FR1, 3, 5, 7, and 10). Response rate during the signal was an inverted-U function of the fixed-ratio requirement. Responding during the safe period and rate of shocks delivered were a direct function of the fixed-ratio requirement. Signal rate (number of times the signal was presented divided by time spent in the safe period) was an inverse function of the fixed-ratio requirement on the warning period. Results are consistent with a cost-benefit analysis of responding in signaled-avoidance procedures. With low response requirement during the signal, rats tended to respond less on the safe period, minimizing effort. Increasing the cost of responding during the signal resulted in that less effort for about the same benefit was attained by responding during the safe period.
\end{abstract}

Key words: negative reinforcement; signaled-avoidance; cost-benefit relation.

Os métodos e teorias adotados nos primeiros estudos sobre o comportamento de esquiva foram elaborados e descritos na tradição pavloviana: um cão era exposto a uma seqüência onde um estímulo originalmente neutro precedia um choque na pata traseira; a resposta "reflexa" ao choque era flexão da pata e a mudança adaptativa era a ocorrência da flexão tão logo o estímulo neutro fosse apresentado (Herrnstein, 1969). Conforme Herrnstein, as sucessivas tentativas de descrever a relação sinal-choque como do tipo CS-US envolviam problemas conceituais que conduziram a tentativas experimentais para solucioná-los. Desse esforço surgiu a primeira teoria bi-fatorial (two-process theory) da esquiva sinalizada, onde componentes respondentes e operantes foram combinados, de modo que a esquiva foi interpretada como subproduto da

1 Endereço: Universidade de Brasília, Instituto de Psicologia, 70910-900, Brasília, DF, Brasil.E-mail: cameschi@unb.br

2 Os autores agradecem ao editor Antônio Pedro de Mello Cruz pela inestimável ajuda na recuperação e composição das figuras do presente trabalho, bem como aos bolsistas de pesquisa Wadar R. Lemes e Sandra R. A. Rocha pelo auxílio na coleta dos dados. fuga do estímulo aversivo condicionado eliciador de "medo" condicionado (Herrnstein, 1969; Cameschi, 1997).

Sidman (1953a, 1953b) elaborou um procedimento de esquiva de operante livre, onde cada resposta produz o adiamento ou cancelamento de choques curtos, inescapáveis e não sinalizados. Em resumo, os choques ocorriam de acordo com um período fixo de tempo (Intervalo choque-choque - SS) e cada resposta produzia um período fixo livre de choques (Intervalo resposta-choque - RS). A explicação da aquisição da resposta de esquiva baseou-se numa teoria da punição de respostas competitivas e, portanto, a esquiva surge como a única resposta possível na situação não punida pelo choque (Sidman, 1953a). Descontente com esta teoria, tendo em vista a velocidade com que muitos sujeitos aprendem a esquiva, tornando implausível que todas as respostas alternativas tenham sido punidas, Sidman (1962a) propôs uma explicação para a aquisição e manutenção da resposta com base apenas nas suas conseqüências: redução na freqüência ou na densidade dos choques. Herrnstein e Hineline (1966) elaboraram um procedimento onde a conseqüência das respostas 
era mudar de alta para baixa a probabilidade do choque, mas poderiam ser seguidas imediatamente por um choque. Portanto, não havia necessariamente atrasos imediatos do choque, mas como no longo prazo a densidade de choque era reduzida pelo responder, a resposta foi modelada e mantida, corroborando a interpretação de Sidman (1962a). Com base nesses resultados, Herrnstein (1969) criticou as especulações das versões da teoria bi-fatorial, sustentando a redução na densidade como condição suficiente e que qualquer estímulo sinalizador do choque adquire funções discriminativas que aceleram a aquisição da resposta de esquiva. Esta função estaria envolvida tanto na esquiva sinalizada onde o sinal é ocasião para a emissão da resposta que o remove e, ao mesmo tempo, cancela ou adia o próximo choque programado, como também nas situações onde a resposta, em vez de remover, produz sinais de período livre de choque, como no estudo de Dinsmoor e Sears (1973).

Em resumo, a história do estudo sobre o controle aversivo do comportamento envolve debates, cuja natureza pode ser, em primeiro lugar, divergências sobre a explicação de certa classe de ação: se o comportamento de esquiva resulta de dois processos (Anger, 1963; Dinsmoor, 1977; Sidman, 1953a) ou de um único (de Villiers, 1974; Herrnstein, 1969; Herrnstein \& Hineline, 1966; Hineline, 1981, 1984; Sidman, 1962a). No centro desta controvérsia está a função dos estímulos imediatos pré e pós-resposta: seriam essencialmente discriminativos ou eliciadores condicionados? Em segundo lugar, temos o debate sobre a utilidade de certas distinções conceituais: por exemplo, considerar desnecessária (Michael, 1975) ou ambígua (Catania, 1999) a distinção entre reforço positivo e negativo. Catania (1999) supõe que, aos poucos, em parte essas divergências teóricas reduziram-se a questões experimentais acerca das condições sob as quais a resposta de esquiva seria adquirida e mantida e as posições divergentes ilustrariam a diferença entre as orientações molecular e molar em análise do comportamento.

Recente publicação, todavia, indica que o debate ainda persiste. Dinsmoor (1977) defendeu sua versão invertida da teoria bi-fatorial, onde a resposta de esquiva é adquirida e mantida por produzir de um período seguro imediato e, portanto, seria caso de reforço positivo. Hineline (1981, 1984) questionou a teoria de Dinsmoor com base em análises empíricas e conceituais das funções dos estímulos nas contingências aversivas. Entretanto, Dinsmoor (2001) segue defendendo uma visão molecular do comportamento de esquiva, baseada no princípio de contigüidade espaçotemporal entre resposta e conseqüência. Além disso, infere que quando não há estímulos-sinais no procedimento, o sinal seguro poderia ser o próprio feedback sensorial produzido pela resposta que, neste caso, estaria sob o controle do sujeito e não do experimentador.

Em resposta a Dinsmoor (2001), Sidman (2001) aceita a sugestão de que a produção do sinal de segurança seria reforço para a esquiva, mas ressalta que na demonstração de que o período seguro pode funcionar como reforço condicionado, sem qualquer término concomitante de um sinal de perigo, o reforço negativo ainda é a base para este estímulo afetar o comportamento. De modo similar, Galizio e Liborio (1995) descrevem que nos estudos sobre os efeitos da remoção da contingência de esquiva contingente a uma resposta alternativa (timeout from avoidance), duas contingências de reforço negativo são comparadas: esquiva e fuga da esquiva. Entretanto, o timeout já foi descrito como caso de reforço positivo (Sidman, 1962b; Verhave, 1962), mas como deriva suas propriedades da fuga da contingência de esquiva, parece mais apropriado considerar como envolvendo contingências de reforço negativo (Galizio \& col., 1995). Courtney e Perone (1992) descrevem que uma instância de timeout from avoidance envolve pelo menos três mudanças na situação experimental: remoção de estímulos associados com a contingência de esquiva, redução na taxa de choques liberados e a suspensão da necessidade de responder associada à contingência de esquiva.

A crítica de Baum (2001) à posição defendida por Dinsmoor (2001) é mais rigorosa que a de Sidman (2001). Segundo Baum, o conflito é paradigmático, pois considera que Dinsmoor defende uma visão molecular do comportamento comprometida com o princípio de contigüidade, superada pela visão molar das contingências entre respostas e conseqüências que aceita e incorpora lacunas temporais entre elas. Hineline (2001) também levanta questões conceituais e filosóficas, como sobre o que funciona como explicação, indicando que em ciência toda explicação conforma-se à descrição de relações funcionais entre variáveis e, portanto, nem toda descrição funciona como explicação. Neste sentido, as variáveis independentes devem estar sob controle experimental e não sob o controle do sujeito, como alega Dinsmoor (2001) para sustentar suas especulações teóricas. Hineline remete aos seus estudos em que atrasos imediatos de eventos aversivos sem redução na densidade são suficientes como reforço negativo (Hineline, 1970 - Experimento 1), mas não se os atrasos imediatos implicam em aumento na densidade (Hineline, 1970 - Experimento 2). Herrnstein (1969) antecipa a análise destes resultados, considerando que os sujeitos, seja respondendo ou deixando de responder, permanecem na situação associada à menor densidade de choque.

Entretanto, Dinsmoor (1977) considera que as situações pré e pós-respostas podem envolver diferentes relações CS-US, e afirma o mesmo com respeito aos resultados de Herrnstein e Hineline (1966), baseado na análise de atrasos médios de choque pós-respostas. Todavia, Hineline (1981, 1984) reitera que esses dados sustentam que as relações temporais imediatas e remotas entre respostas e remoção de eventos aversivos são negativamente reforçadoras, sendo desnecessário ir além do nível descritivo para reconhecer os processos molar e molecular no controle do comportamento. Com base nisto, Hineline $(1984,2001)$ sugere como mais produtivo avançar além da distinção molar-molecular em direção a análises multi-escalares. Por outro lado, Baron e Perone (2001) julgam a teoria de Dinsmoor (2001) mais eficaz para explicar o comportamento de esquiva por sua ênfase no intercâmbio entre contingências respondentes e operantes, encorajando a análise dos estímulos mediadores das conseqüências molares e, assim, incorporando o controle por eventos locais tanto quanto dos eventos temporalmente mais remotos, improváveis ou cumulativos. Entretanto, os mesmos autores criticam o valor heurístico das teorias em confronto, pois muito pouca pesquisa sobre esquiva foi realizada desde os anos 1970, de modo que "se um critério para julgar uma boa teoria é sua 'fertilidade', nenhuma destas 
visões da esquiva parece se ajustar a ele muito bem" (Baron \& Perone, 2001, p. 360).

Com respeito às divergências teóricas, uma das questões importantes diz respeito ao papel do sinal que precede a apresentação de um choque, nos procedimentos de esquiva sinalizada. Alguns autores defendem uma função eliciadora condicionada de estados corporais condicionados (Anger, 1963; Dinsmoor, 1977, 2001), outros enfatizam funções discriminativas (Herrnstein, 1969; Herrnstein \& Hineline, 1966), mas análises mais amplas reconhecem que complexas interações operantes-respondentes podem surgir nas contingências aversivas, integrando as relações entre os estímulos antecedentes e conseqüentes ao comportamento (Hineline, 1981, 1984, 2001).

Skinner $(1938,1953,1969)$ definiu a função discriminativa de um estímulo como ocasião que torna uma resposta mais provável de ocorrer. Em procedimentos de esquiva sinalizada, tipicamente um período nunca correlacionado com choque - período seguro - precede um outro correlacionado com o choque - período de aviso. A probabilidade de ocorrer resposta em um ou em outro período pode ser maior ou menor, dependendo das contingências de reforço negativo programadas para cada período. A resposta de esquiva pode não ser tanto função de fatores momentâneos conforme implica a função eliciadora, mas sim, em grande parte, sua probabilidade parece ser função da interação entre processos moleculares e molares da situação (Hineline, 1981, 1984, 2001).

Todorov, Carvalho e Menandro (1977) comentam como plausível se argumentar contra uma separação arbitrária entre as fases de aquisição e de desempenhos posteriores no condicionamento de esquiva. Mas, sugerem que no procedimento de Sidman (1953a) esta separação parece razoável nos seguintes aspectos: (a) uma vez que a estabilidade tenha sido atingida, as variáveis importantes para determinar a velocidade da aquisição da resposta de esquiva podem ter pouco ou nenhum efeito sobre as taxas de resposta e de choques recebidos (por exemplo, duração do intervalo SS e intensidade de choque); (b) o procedimento de Sidman (1953a, 1953b) combina três fatores e cada qual sozinho poderia ser responsável pela aquisição da resposta de esquiva: responder resulta em redução na freqüência de choque, em período livre de choque e em nenhum choque logo após a resposta. Depois de se atingir a estabilidade no responder, somente mudanças nas relações temporais entre respostas e choques claramente afetam a taxa de respostas; (c) como confunde a influência destes três fatores, este procedimento torna-se inadequado para a análise da aquisição do comportamento de esquiva. Entretanto, as relações temporais entre respostas e choques que ele permite tornam seu uso uma ferramenta conveniente para o estudo do tempo como estímulo discriminativo no controle do comportamento quando este atinge o estado estável (Todorov \& cols., 1977).

Para estudar as relações entre a intensidade do choque e as taxas de respostas em estado estável, de Souza, de Moraes e Todorov (1984) submeteram ratos a uma contingência de esquiva sinalizada de operante livre. Ao longo do experimento a intensidade do choque variou de 0,1 a $8,0 \mathrm{~mA}$, mantendo constante sua duração em 200 milisegundos. Os resultados indicam: (a) um efeito tudo-ou-nada da intensidade do choque sobre as taxas de respostas e de choques, na porcentagem de choques evitados e na frequiência da resposta durante o estímulo pré-choque; e (b) nenhum efeito sistemático da intensidade de choque sobre o controle de estímulos, seja medido pela porcentagem de apresentações de estímulos seguidas por respostas ou pela porcentagem de respostas que ocorreram durante os estímulos pré-choque. Portanto, segundo de Souza e cols. (1984), para cada sujeito há uma intensidade de choque mínima necessária para estabelecer e manter a resposta de esquiva, de modo que aumentos na intensidade acima deste valor mínimo têm pouco ou nenhum efeito sobre a taxa de respostas, exceto o efeito deletério geral do comportamento sob intensidade de choque muito além deste limite. Estes resultados confirmam o primeiro aspecto apontado por Todorov e cols. (1977) acima e divergem das suposições presentes na literatura sobre esquiva, onde geralmente a taxa de resposta é descrita como uma função monotônica direta e negativamente acelerada da intensidade de choque (de Souza \& cols., 1984).

Portanto, para evitar os efeitos de variáveis que afetam o processo de aquisição e/ou as fases de transição do comportamento, o presente estudo considerou somente os resultados em estados estáveis e teve como objetivo a análise da relação custo-benefício do responder em procedimento de esquiva sinalizada, onde o custo para evitar o choque aumentou progressivamente durante o período de aviso, enquanto foi mantido constante durante o período seguro.

\section{Método}

\section{Sujeitos}

Foram utilizados seis ratos albinos Wistar, sem história experimental anterior, provenientes do Biotério Central da Universidade de Brasília. Fora das sessões experimentais, os sujeitos eram mantidos em gaiolas-viveiros individuais, com água e alimento disponíveis o tempo todo.

\section{Equipamento}

Foi utilizada uma câmara experimental para estudos de controle aversivo do comportamento de ratos. A câmara media 24 x 16,5 x 21,5 cm, contendo um painel de resposta de 7 x $5 \mathrm{~cm}$, colocado a $9,5 \mathrm{~cm}$ acima do assoalho e fixado a uma das paredes, avançando para o interior da câmara formando um angulo de 45 graus. $\mathrm{O}$ assoalho era composto de 16 barras metálicas de $2 \mathrm{~mm}$ de diâmetro, separadas entre si por $1,5 \mathrm{~cm}$. As barras metálicas estavam ligadas por fios condutores a um gerador de choques Grason-Stadler (EUA), modelo E $600 \mathrm{~B}$, com scrambler, isto é, misturador de polaridades. Havia no interior da câmara um sistema emissor de estímulos-sinais, luminoso e sonoro, composto por uma lâmpada e sons gerados por um alto-falante. Para o controle de ruídos estranhos ao experimento, a câmara experimental estava situada dentro de uma câmara de isolamento acústico e visual Grason-Stadler (EUA), modelo E 3125, série A-300, localizada em sala experimental acusticamente isolada. A programação e o registro dos eventos eram realizados por circuitos eletromecânicos convencionais, situados em sala adjacente ao ambiente experimental. 


\section{Procedimento}

Depois da resposta de pressão ao painel ser modelada, os sujeitos foram expostos a uma contingência de esquiva sinalizada com tentativas de 30 segundos, divididos em dois períodos. Durante 20 segundos vigorava o período seguro (PS), durante o qual a câmara experimental permanecia no escuro, e era seguido pelo período de aviso (PA), onde durante 10 segundos uma combinação de som e luz precedia a ocorrência de um choque de 0,5 segundo de duração e 1 $\mathrm{mA}$ de intensidade. Nesta fase, uma resposta durante o PS reiniciava este período, bem como uma resposta durante o PA eliminava o sinal e evitava o choque, restabelecendo as condições do PS. Nas fases subsequientes, o responder durante o PA foi reforçado de acordo com um esquema de Razão Fixa (FR) crescente, na seguinte ordem: FR 2, FR 3, FR 5, FR 7 e FR 10. Cada fase vigorou durante 15 sessões diárias de 60 minutos de duração. Dois dos sujeitos (ER-5 e ER-6) retornaram a FR 1 e FR 3 e permaneceram nestas condições durante 15 e 24 sessões, respectivamente.

\section{Resultados}

As Figuras 1 e 2 mostram as taxas de respostas emitidas durante o período seguro e período de aviso, em função do número de respostas requerido durante o período de aviso. Analisando as Figuras de modo comparativo, pode-se observar na Figura 1 que os desempenhos dos sujeitos ER-1 e ER-5 mostram nitidamente decréscimos nas taxas à medida que aumenta o requisito de razão durante o sinal, enquanto que os dados do sujeito ER-6 revelam este declínio a partir de FR 7.

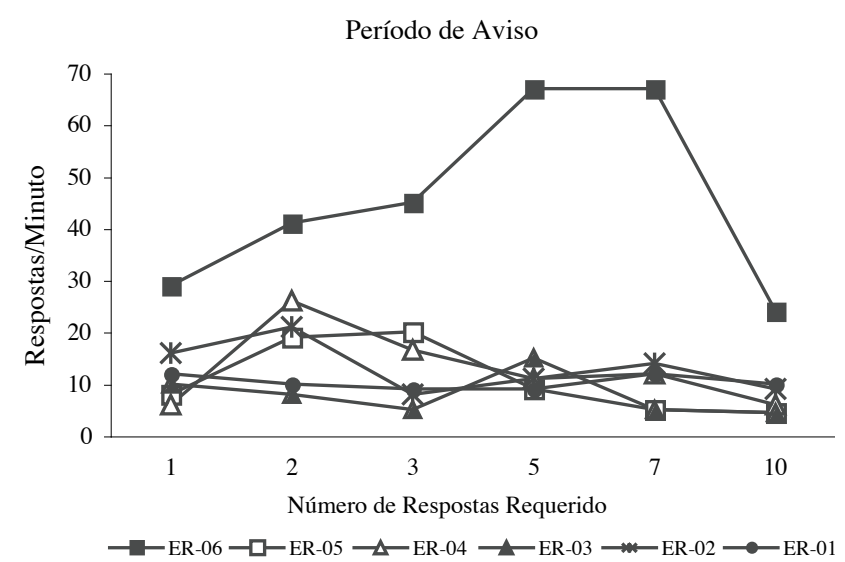

Figura 1. Taxas de respostas no período de aviso em função do número de respostas requerido no período de aviso.

Em contrapartida, pode-se observar na Figura 2 uma nítida tendência crescente das taxas, durante o período seguro, à medida que aumenta o valor da FR contingente ao período de aviso.

Os dados de todos os sujeitos foram agrupados, e as taxas médias do grupo em função do requisito de razão requerido são mostradas nas Figura 3 e 4, que representam, respectivamente, os desempenhos durante o período de aviso e período seguro.

Pode-se observar na Figura 3 que a taxa do sujeito ER-6 mascara a tendência geral de declínio das taxas durante o

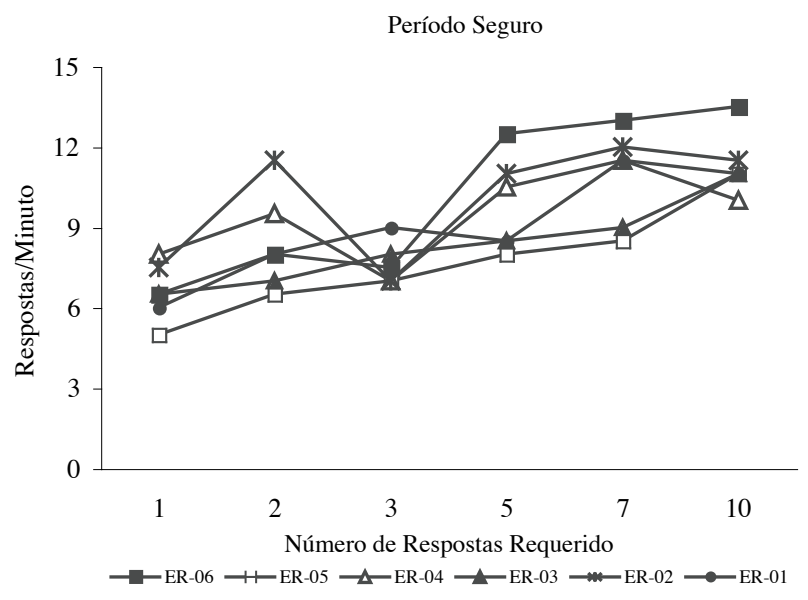

Figura 2. Taxas de respostas no período seguro em função do número de respostas requerido no período de aviso.

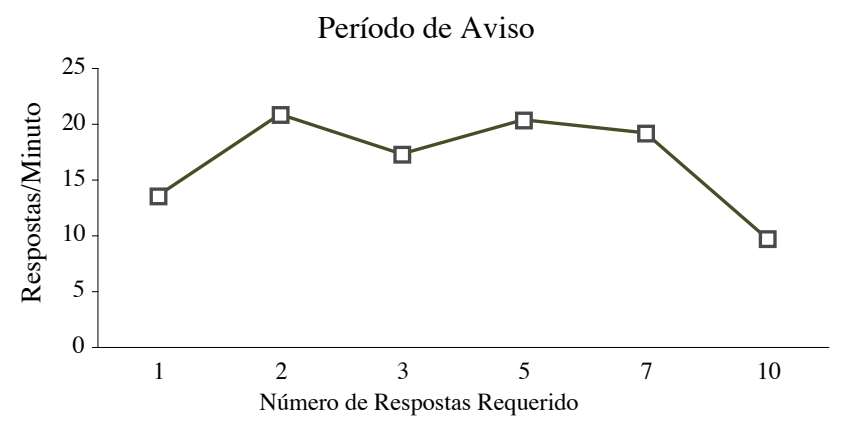

Figura 3. Médias das taxas de respostas no período de aviso em função do número de respostas requerido no período de aviso.

período de aviso, enquanto a Figura 4 mostra que os dados do grupo refletem as tendências crescentes das taxas durante o período seguro, conforme acima foi apontado.

A Figura 5 mostra a taxa de apresentação de estímulos de aviso em função do valor da FR requerida. Pode-se verificar que à medida que FR aumenta durante o período de aviso, diminui a taxa de apresentação do estímulo-sinal, o que reflete também o aumento nas taxas de respostas durante o período seguro, cuja conseqüência era a manutenção deste período.

Finalmente, as taxas de choques aumentaram em função do aumento da FR durante o período de aviso, sugerindo que quando ocorria o período seguro, as razões fixas maiores não eram completadas e, portanto, os choques eram liberados mais freqüentemente.

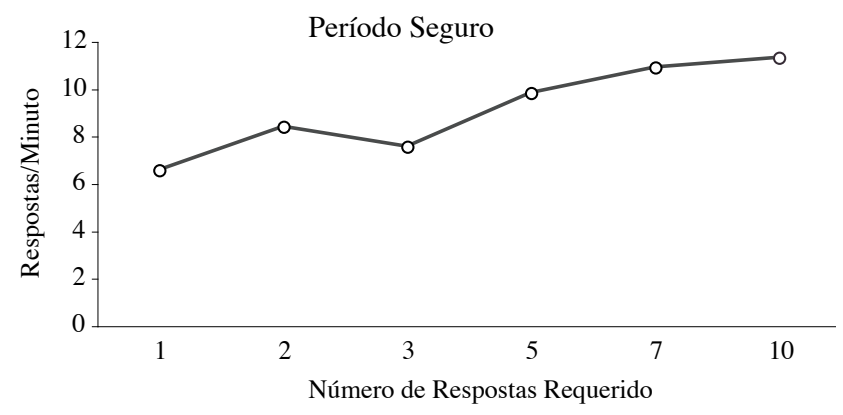

Figura 4. Médias das taxas de respostas no período seguro em função de número de respostas requerido no período de aviso. 


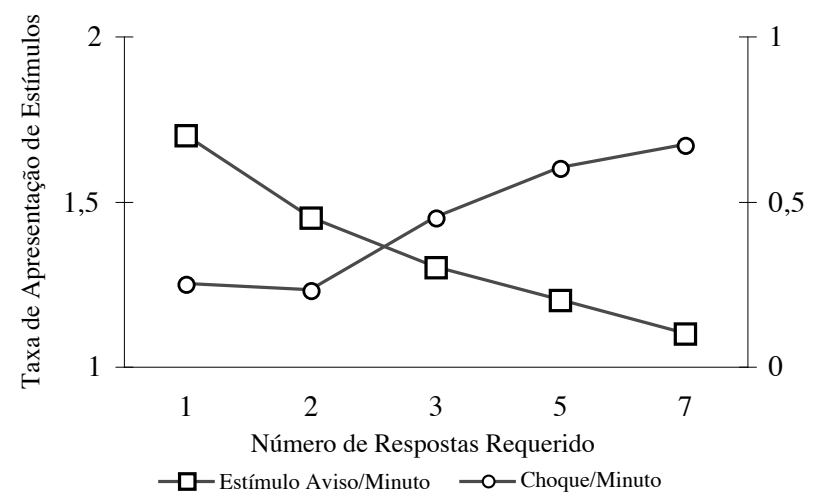

Figura 5. Taxas de apresentações de estímulos em função de número de respostas requerido no período de aviso.

\section{Discussão}

Os resultados estão consistentes com uma análise custobenefício do responder em procedimentos de esquiva sinalizada. Com baixa exigência de respostas durante o aviso, os sujeitos tendem a responder menos no período seguro, minimizando o esforço, isto é, evitando o mesmo número de choques com menos respostas. Com o aumento do custo do responder durante o período de aviso (aumento na FR), passar a responder durante o período seguro reduziu o esforço para obter o mesmo benefício.

A mudança do responder do período de aviso para o período seguro implica que além de evitar o choque, os sujeitos evitavam também a situação aversiva adicionada ao período de aviso, ou seja, o maior número de respostas requerido para o reforço negativo. Esta mudança parece similar à observada por Mellitz, Hineline, Whitehouse e Laurence (1983) que, em estudo em que a resposta de esquiva podia ocorrer em uma ou outra barra, após a aquisição identificaram o desenvolvimento de uma preferência por responder em uma das alternativas. Então, introduziram um esquema de reforço negativo conjugado na alternativa não preferida: além de cada resposta de esquiva no lado não preferido produzir o intervalo RS programado, produzia também redução de 1 min na duração da sessão. Os sujeitos passaram a responder mais no lado do esquema conjugado em várias reversões entre a barra $\mathrm{A}$ e $\mathrm{B}$, reduzindo não somente a densidade de choques, mas também cerca de $80 \%$ da duração da sessão de 152 minutos (Mellitz \& cols., 1983).

No estudo de Courtney e Perone (1992), após modelagem e treino em esquiva de Sidman ( $\mathrm{SS}=5 \mathrm{~s}$ e RS $=30 \mathrm{~s}$ ), ratos foram expostos a um esquema concorrente esquiva-timeout, onde foi programada uma discriminação de time-in e timeout, por meio de um esquema múltiplo de três componentes que se alternavam: em dois dos componentes ocorriam ciclos variáveis independentes de programação de choques com média de $30 \mathrm{~s}$ (VC $30 \mathrm{~s}$ ), em que a primeira resposta na barra de esquiva cancelava o próximo choque programado e respostas adicionais no mesmo ciclo não tinham conseqüências programadas. Um destes componentes era acompanhado por iluminação constante e o outro por iluminação fragmentária; no terceiro componente - timeout - a iluminação era desligada. Inicialmente, cada pressão na barra timeout produzia 5 min de remoção da contingência de esquiva que, depois, foi reduzido para $2 \mathrm{~min}$, enquanto o esquema para produzir o timeout mudou para VI $15 \mathrm{~s}$ até atingir seu valor terminal, VI $45 \mathrm{~s}$. Os autores verificaram que o responder na barra timeout foi relativamente insensível à redução na taxa de choques, e que um fator mais potente seria a redução temporária do esforço associada à suspensão dos esquemas VC de eliminação dos choques. Ou seja, ao produzir o timeout os ratos escapam da contingência que exige responder contínuo e inicia-se uma situação alternativa sem requerimento de resposta. Os autores concluem que os resultados apóiam a sugestão de Hineline (1984) de que a aversividade de uma situação comportamental pode depender de uma faixa de eventos (ou relações entre eventos), além do estímulo primário como o choque (Courtney \& Perone, 1992).

A resistência à extinção da pressão à barra mantida por timeout da esquiva foi examinada por Galízio (1999 - Experimento 1), onde ratos foram expostos a um esquema concorrente onde: (a) cada resposta em uma das barras produzia breve término de ruído branco (feedback da resposta $=0.5 \mathrm{~s})$ e adiavam os choques $(\mathrm{SS}=5 \mathrm{~s} ; \mathrm{RS}=30 \mathrm{~s})$; (b) inicialmente, cada resposta em outra barra produzia 5 min de timeout sinalizado pela retração da barra, remoção do ruído branco, pelo acender ou apagar a luz da câmara e suspensão do choque. Após estabilizar a resposta nesta barra, a duração do timeout foi reduzida a 2 min e o esquema foi gradualmente modificado para VR 15; (c) dois tipos de extinção foram estudados: (1) a extinção da resposta na barra timeout envolveu a suspensão deste, mantendo os estímulos produzidos em VR 15 e inalterada a contingência na outra barra; (2) o gerador de choques foi desligado, enquanto todos os outros aspectos do procedimento permaneceram como na linha de base. Para três sujeitos, a luz ambiente acendia e apagava, respectivamente, no início e fim de cada sessão de 2 horas; para outro sujeito, era o inverso. Os resultados mostram que taxas de respostas estáveis foram mantidas em ambas as barras, sendo que as taxas médias de respostas na barra-timeout foram sempre maiores do que as taxas na barra-esquiva. A suspensão do reforço na barra-timeout produziu rápida extinção: em cerca de uma hora todos os quatro sujeitos reduziram a taxa a níveis muito baixos. Em contraste, a suspensão do choque teve pouco efeito nas taxas de resposta na barra-timeout, enquanto as taxas de resposta de esquiva de alguns sujeitos diminuíram ao longo das sessões (Galízio, 1999).

A persistência do responder na barra-timeout na condição sem choques conduziu ao estudo do curso da extinção associada com a omissão do choque, conforme o experimento 1 , ao longo de muitas sessões (Galizio, 1999 - Experimento 2). Participaram como sujeitos quatro ratos experientes em esquemas concorrentes esquiva de Sidman e timeout da esquiva em VR 15, dois do Experimento I e outros dois com histórias de condicionamento comparáveis. As taxas de respostas de esquiva diminuíram relativamente rápido ao longo de cinco a 10 sessões, enquanto o declínio das taxas timeout foi mais lento, sendo que dois sujeitos permaneceram respondendo ao nível da linha de base por mais de 20 sessões de extinção. Estes resultados mostram que o responder mantido por reforço negativo pode ser altamente resistente à extinção, sendo a persistência do responder na barra timeout difícil de ser explicada pelas teorias atuais. Conforme Galízio, este resultado não apoia: (a) a suposição de Courtney e Perone 
(1992) de que reduções locais na freqüência de respostas são a base das propriedades reforçadoras do timeout, portanto a persistência deste responder durante a extinção sugere outros determinantes além da redução do esforço em responder; (b) a ênfase na redução na densidade defendida por Herrnstein e Hineline (1966); (c) as teorias cognitivas que enfatizam a expectativa do choque como subjacente à esquiva, conforme proposto por Seligman e Johnston (1973); (d) as predições das teorias bi-fatorias tradicionais de que tanto as respostas de esquiva e timeout são mantidas pelo término de estímulos emparelhados com choques e que as durações da extinção seriam proximamente relacionadas. Em conseqüência, o autor pergunta: "Por que o estímulo timeout retém suas propriedades reforçadoras quando a densidade de choque é zero e há insuficiente "medo" ou "expectativa" do choque para motivar a esquiva?” (Galizio, 1999, p. 10).

Portanto, parece que as teorias disponíveis não explicam os processos resultantes do timeout da esquiva, assim como ainda debatem sobre qual o reforço para o comportamento de esquiva. Entretanto, as interações organismo-ambiente observadas no presente estudo, como aquelas em Mellitz e cols. (1983), Courtney e Perone (1992) e Galizio (1999), implicam que o comportamento seja afetado por grupos de variáveis que atuam como um conjunto e oferecem apoio às sugestões de Baum (2001) e Hineline (1981, 1984, 2001). Ou seja, a abordagem molar parece descrever melhor a integração entre respostas e consequiências, incluindo contingências com lacunas temporais e espaciais entre elas. No presente estudo, as transições das taxas de resposta entre os períodos de aviso e seguro em função das relações custo-benefício embutidas nas contingências sugerem que estes períodos são situações que alteram a probabilidade da resposta porque adquirem funções de estímulos discriminativos (Herrnstein, 1969; Hineline, 1981; Skinner, 1938, 1953, 1969), sendo menos plausíveis que atuem como eliciadores condicionados de estados corporais condicionados, cujas funções mediadoras são defendidas pelas diversas versões da teoria bi-fatorial (Hineline, 1970 - Experimento 3).

Em estudo que investigou o controle de estímulos em esquiva de operante livre, de Souza, de Morais e Todorov (1992) testaram os efeitos de estímulos visuais (luz-escuro) e auditivos (som-silêncio) associados à liberação de choque em esquiva sinalizada. Um grupo com sete ratos foi exposto a duas condições S1S2 pré-choque contrabalançadas: (1) luzescuro-choque; e (2) escuro-luz-choque. Outro grupo com 18 ratos foi exposto a quatro destas condições: (1) silênciosom-choque, com luz da câmara apagada; (2) som(escuro)silêncio(luz)-choque; (3) silêncio-som-choque, com luz da câmara acesa; (4) silêncio(luz)-som-luz vermelha(+ luz da câmara). Na linha de base a duração do $\mathrm{S} 1$ era 15 s e do S2 era $10 \mathrm{~s}$, uma resposta durante o $\mathrm{S} 1$ reiniciava a tentativa enquanto uma resposta durante o $\mathrm{S} 2$ removia este sinal e cancelava o choque na tentativa. Portanto, sem emissão de respostas o intervalo entre choques (S1S2) era de $25 \mathrm{~s}$.

Dois tipos de sondagens do controle de estímulo foram feitos: (a) na primeira metade de uma sessão de $60 \mathrm{~min}$ permanecia constante a duração do S1 (15 s) e na segunda metade ela variou de 2 a 30 s (VT 30 s); (b) as sessões de teste foram divididas em seis períodos de $10 \mathrm{~min}$, onde a linha de base se alternava com períodos com nova duração do S1
$(15,5,15,150,15,30 \mathrm{~s})$, portanto com quatro sondagens. Os procedimentos de sondagem mostraram que o controle exercido pelo estímulo auditivo foi melhor do que o visual e não dependeu de qualquer processo de discriminação temporal subjacente, que pode ter tido um papel mais importante no desempenho do grupo visual. Os autores concluem que o estudo mostra que os estímulos visuais podem exercer controle discriminativo do comportamento de esquiva de ratos, mas os estímulos auditivos são melhores do ponto de vista de uma análise custo-benefício, pois o grupo auditivo recebeu poucos choques nas sessões de linha de base com poucas respostas durante o período seguro e mostra menos dependência de discriminação temporal nas sessões de sondagem (de Souza \& cols., 1992). Os resultados do presente estudo também confirmam funções discriminativas do sinal, ao estabelecer a melhor ocasião para a emissão da resposta em função da relação custo-benefício.

\section{Referências}

Anger, D. (1963). The role of temporal discriminations in the reinforcement of Sidman avoidance behavior. Journal of the Experimental Analysis of Behavior, 6, 477-506.

Baron, A. \& Perone, M. (2001). Explaining avoidance: Two factors are still better than one [Reply to Dinsmoor (2001]. Journal of the Experimental Analysis of Behavior, 75, 357-361.

Baum, W.N. (2001). Molar versus molecular as a paradigm clash [Reply to Dinsmoor, 2001]. Journal of the Experimental Analysis of Behavior, 75, 338-341.

Cameschi, C.E. (1997). Comportamento de esquiva: teorias e controvérsias. Psicologia: Teoria e Pesquisa, 13(1), 143-152.

Catania, A.C. (1999). Aprendizagem: comportamento, linguageme cognição. Trad. e revisão de Daisy das Graças de Souza. Porto Alegre: Artmed Editora.

Courtney, K. \& Perone, M. (1992). Reduction in shock frequency and response effort as factors in reinforcemente by timeout from avoidance. Journal of the Experimental Analysis of Behavior, 58, 485-496.

de Souza, D.G., de Moraes, A.B.A. \& Todorov. J.C. (1984). Shock intensity and signaled avoidance responding. Journal of the Experimental Analysis of Behavior, 42, 67-74.

de Souza, D.G., de Moraes, A.B.A. \& Todorov. J. C. (1992). Probing stimulus control in free-operant avoidance. The Psychological Record, 42, 41-55.

de Villiers, P.A. (1974). The law of effect and avoidance: a quantitative relationship between response rate and shock-frequency reduction. Journal of the Experimental Analysis of Behavior, 21, 223-235.

Dinsmoor, J.A. (1977). Escape, avoidance, punishment: Where do we stand? Journal of the Experimental Analysis of Behavior, 28, 83-95.

Dinsmoor, J.A. (2001). Stimuli inevitably generated by behavior that avoids eletric shock are inherently reinforcing. Journal of the Experimental Analysis of Behavior, 75, 311-333.

Dinsmoor, J.A. \& Sears, G.W. (1973). Control of avoidance by a response-produced stimulus. Learning and Motivation, 4, 284-293.

Galizio, M. (1999). Extinction of responding maintained by timeout from avoidance. Journal of the Experimental Analysis of Behavior, 71, 1-11. 
Galizio, M. \& Liborio, M.O. (1995). The effects of cocaine on behavior maintained by timeout from avoidance. Journal of the Experimental Analysis of Behavior, 63, 19-32.

Herrnstein, R.J. (1969). Method and theory in the study of avoidance. Psychological Review, 76, 49-69.

Herrnstein, R.J. \& Hineline, P.N. (1966). Negative reinforcement as shock-frequency reduction. Journal of the Experimental Analysis of Behavior, 9, 421-430.

Hineline, P.N. (1970). Negative reiforcement without shock-frequency reduction. Journal of the Experimental Analysis of Behavior, 14, 259-268.

Hineline, P.N. (1981). The several roles of stimuli in negative reinforcement. Em P. Harzen \& M.D. Zeiler (Orgs.), Advances in analysis of behavior (Vol. 2). Predictability, correlation and contiguity (pp. 203-246). New York: Wiley.

Hineline, P.N. (1984). Aversive control: A separate domain? Journal of the Experimental Analysis of Behavior, 42, 495-509.

Hineline, P.N. (2001). Beyond the molar-molecular distinction: We need multiscaled analysis [Reply to Dinsmoor (2001)]. Journal of the Experimental Analysis of Behavior, 75, 342-347.

Mellitz, M., Hineline, P.N., Whitehouse, W.G., \& Laurence, M.T. (1983). Duration-reduction of avoidance sessions as negative reinforcement. Journal of the Experimental Analysis of Behavior, 40, 57-67.

Michael, J. (1975). Positive and negative reinforcement, a distinction that is no longer necessary: Or a better way to talk about bad things. Behaviorism, 3, 33-44.

Seligman, M.E.R. \& Johnston, J.C. (1973). A cognitive theory of avoidance learning. Em F.J. McGuigan \& D.B. Lumsden
(Orgs.), Contemporary approaches to conditioning and learning (pp. 69-110). Washington, DC: V.H. Winston \& Sons.

Sidman, M. (1953a). Avoidance conditioning with brief shock and no extereoceptive warning signal. Science, 118, 157-158.

Sidman, M. (1953b). Two temporal parameters of the maintenance of avoidance behavior by white rat. Journal of Comparative and Physiological Psychology, 46, 253-261.

Sidman, M. (1962a). Reduction of shock frequency as reinforcement for avoidance behavior. Journal of the Experimental Analysis of Behavior, 5, 247-257.

Sidman, M. (1962b). Time out from avoidance as a reinforcer: a study of response interaction. Journal of the Experimental Analysis of Behavior, 5, 423-434.

Sidman, M. (2001). Safe periods both explain and need explaining (Reply to Dinsmoor, 2001). Journal of the Experimental Analysis of Behavior, 75, 335-338.

Skinner, B.F. (1938). The behavior of organisms. New York: Appleton-Century-Crofts.

Skinner, B.F. (1953). Science and human behavior. New York: Mcmillan.

Skinner, B.F. (1969). Contingencies of reinforcement. New York: Apletton-Century-Crofts.

Todorov, J.C., Carvalho, L.C.F. \& Menandro, P.R.M. (1977). The standard Sidman avoidance procedure as a temporal differentiation schedule. Revista Mexicana de Análisis de la Conducta, $3(2), 151-160$.

Verhave, T. (1962). The functional properties of a timeout from an avoidance schedule. Journal of the Experimental Analysis of Behavior, 5, 391-422. 


\section{Princípios Normativos Para Publicação em Psicologia: Teoria e Pesquisa}

\section{Permissão para reprodução}

Recomenda-se evitar a reprodução de material já publicado, a menos que seja extremamente necessária.

Se o manuscrito submetido contém reprodução de material já publicado - figura, ou tabela, ou citação de texto que exceda 500 palavras - o autor deve encaminhar ao editor uma fotocópia da permissão escrita para essa reprodução, em acordo com as normas de direitos autorais da publicação original. Essa exigência deve ser cumprida inclusive no caso de reprodução de material publicado em Psicologia: Teoria e Pesquisa, a menos que seja material do próprio autor.

No caso de reprodução de material do próprio autor, a comprovação de direito de reprodução pode ser dispensada apenas se for material publicado originalmente em Psicologia: Teoria e Pesquisa.

No caso de modificação de uma figura, ou adaptação de modelos ou diagramas, recomenda-se que o autor encaminhe a documentação da fonte original que permita ao Conselho Editorial avaliar a adaptação feita.

Em sua maior parte, o conteúdo deste texto foi traduzido e adaptado do Manual de Publicação da APA (APA, 1994). 